\title{
Project New Orion: Pulsed Nuclear Space Propulsion Using Photofission Activated by Ultra-Intense Laser
}

\author{
Robert LeMoyne1, Timothy Mastroianni² \\ ${ }^{1}$ Independent (Senior Member AIAA \& Senior Member IEEE), Running Springs, California, USA \\ ${ }^{2}$ Independent, Pittsburgh, Pennsylvania, USA \\ Email: rlemoyne07@gmail.com
}

Received 25 December 2015; accepted 6 April 2016; published 13 April 2016

\begin{abstract}
Project New Orion entails a pulsed nuclear space propulsion system that utilizes photofission through the implementation of an ultra-intense laser. The historical origins derive from the endeavors of Project Orion, which utilized thermonuclear devices to impart a considerable velocity increment on the respective spacecraft. The shear magnitude of Project Orion significantly detracts from the likelihood of progressive research development testing and evaluation. Project New Orion incorporates a more feasible pathway for the progressive research development testing and evaluation of the pulsed nuclear space propulsion system. Photofission through the application of an ultra-intense laser enables a much more controllable and scalable nuclear yield. The energy source for the ultra-intense laser is derived from a first stage liquid hydrogen and liquid oxygen chemical propulsion system. A portion of the thermal/kinetic energy of the rocket propulsive fluid is converted to electrical energy through a magneto-hydrodynamic generator with cryogenic propellant densification for facilitating the integral superconducting magnets. Fundamental analysis of Project New Orion demonstrates the capacity to impart a meaningful velocity increment through ultra-intense laser derived photofission on a small spacecraft.
\end{abstract}

\section{Keywords}

Project Orion, Pulsed Nuclear Space Propulsion, Photofission, Nuclear Fission, Ultra-Intense Laser, Magneto-Hydrodynamic Generator, Cryogenic Propellant Densification

\section{Introduction}

Non-chemical space propulsion offers inherent advantages from a performance perspective with a highly economical means of imparting a considerable velocity increment on the respective spacecraft. The domain of non-chemical propulsion spans the realm of nuclear reactions, such as antimatter annihilation and photofission. Antimatter and photofission pulsed space propulsion configurations have been proposed and conceptualized by LeMoyne and Mastroianni. The foundation to the initiation of these two types of non-chemical propulsion is the 
operation of an ultra-intense laser system [1] [2].

The antimatter and photofission pulsed space propulsion systems using an ultra-intense laser system incorporated a thermal energy threshold pulsed space propulsion configuration. Upon the pulsed space propulsion system reaching a thermal threshold from antimatter annihilation or photofission, a prescribed pulse of propulsive fluid, such as hydrogen is released. The activation through an ultra-intense laser system enables a decoupling between the location of the propulsion system and laser energy source [1] [2].

Another type of pulsed space propulsion incorporates a thermonuclear denotation. Project Orion characterized this concept during the 1960's. In essence, Project Orion utilized a thermonuclear detonation as a means of imparting a substantial velocity increment upon the prescribed spacecraft [3]-[5].

However, the shear magnitude of Project Orion may intrinsically decrement the feasibility of developing nuclear pulsed space propulsion. As Project Orion incorporates a thermonuclear detonation in a space propulsion context, the thermonuclear device must be first transferred from a terrestrial environment to a space orbit. The safety risk is highly paramount. Even aerial transport of operational nuclear devices even within a nation's sovereign airspace is inherently dangerous as the potential for unintended nuclear detonations are of historically sobering relevance [6]. The safe distance between the Earth in general and a sizeable nuclear detonation on the scale of a thermonuclear weapon is also a debatable topic.

Project New Orion is proposed as an operationally feasible mechanism for the progressive test and evaluation of nuclear derived pulsed space propulsion. Instead of utilizing a large magnitude thermonuclear detonation to provide a velocity increment, photofission by means of an ultra-intense laser is applied. A notable advantage of photofission is the ultra-intense laser derived activation and scalable yield [2].

Project New Orion is derived from the synthesis of multiple high technology perspectives. The propulsive velocity increment is derived from a photofission event based on activation from an ultra-intense laser. The Project New Orion pulsed nuclear space propulsion system will constitute the second stage of a space launch vehicle using liquid hydrogen and liquid oxygen propulsion. The first stage will facilitate the energy source for the ultra-intense laser system. The ultra-intense laser energy will be converted from propulsive fluid thermal/ kinetic energy to electrical energy for the ultra-intense laser through magneto-hydrodynamics. The superconducting magnets for providing magneto-hydrodynamic energy conversion will utilize the benefits of cryogenic propellant densification. With the Project New Orion conceptual architecture elucidated, the objective is to demonstrate the fundamental performance analysis of Project New Orion.

\section{Background}

\subsection{Project Orion: A Historical Perspective}

Project Orion stems from the research, development, testing, and evaluation endeavors of the United States of America during the Cold War Era [3] [4]. Preliminary interest regarding the incorporation of nuclear detonations as a mechanism for imparting a considerable velocity increment transpired during the late 1940's to mid 1950's. Beginning in the later 1950's fundamental analytical and experimental studies elucidated basic engineering and physical issues for pulsed space propulsion [3].

This pulsed nuclear propulsion strategy was presented as an alternative to traditional chemical propulsion configurations, such as the Saturn V launch vehicles. The preliminary objective was to transfer spacecraft to Venus and Mars even by the late 1960's [4]. The advantage of the Project Orion configuration is the considerable energy density utilized for imparting kinetic energy to the spacecraft, such as through a thermonuclear detonation [3] [4].

The Project Orion incorporates a series of pulsed thermonuclear detonations that propel the spacecraft with a considerable velocity increment [3]-[5]. A chemical analogy may be provided for contrast to nuclear pulsed propulsion. In essence, nuclear pulsed propulsion on a chemical scale is similar to the ignition of a gunpowder charge for a rifle cartridge. Based on the characteristics of the rifle, the chemical energy of the gunpowder charge imparts a considerable amount of kinetic energy on the respective bullet.

The features of the Project Orion incorporate a mechanism for absorbing a portion of the thermonuclear detonation energy for the kinetic energy increment to the spacecraft. This mechanism for converting the energy of the thermonuclear detonation to spacecraft kinetic energy increment is achieved through a through a nozzle-like pusher that literally catches a portion of the thermonuclear detonation. The geometric configuration for the nozzle-like pusher varies from hemispherical to disk morphologies [3]-[5]. 
The inherent nature of a thermonuclear detonation encompasses considerable thermal energy in tandem with kinetic energy. Therefore, an ablation system is amended to the Project Orion design. Between the nozzle-like pusher and payload a medium to ameliorate the acceleration load is imperative. A shock absorbing mechanism is applied between the nozzle-like pusher and payload aspect of the Project Orion configuration to reduce the acceleration load on the payload section. Thermal loads incident on the nozzle-like pusher of a Project Orion configuration necessitate the incorporation of ablation techniques and heat sinks to sustain the payload section of the spacecraft [3]-[5].

The pulsed nuclear propulsion envisioned in Project Orion incorporates thermonuclear detonations on the scale of a megaton yield [3]-[5]. Attaining a spacecraft configuration on the scale of such a magnitude presents a daunting endeavor. The capacity to institute a representative and meaningful subscale application for test and evaluation may even be preclusive based on the shear magnitude of the concept.

Other issues with a megaton yield nuclear pulsed propulsion system may inhibit forward research, development, test, and evaluation. Safely transporting a thermonuclear device to space orbit and finding a suitable location for detonation may not be feasible for the consent of the international community. Progressively transporting and assembling materials associated with Project Orion, such as the nozzle-like pusher and shock absorption mechanism, may alone be a difficult task to realistically satisfy in consideration of current launch vehicle technologies.

Project New Orion promotes a potentially more feasible pathway for the progressive evolution of research, development, test, and evaluation for nuclear pulsed propulsion. Rather than applied thermonuclear devices on the scale of a megaton yield Project New Orion incorporates photofission through the use of an ultra-intense laser. From a safety perspective photofission occurs with the initiation of an incident ultra-intense laser pulse, therefore decoupling the energy source and nuclear pulsed propulsion. Also, photofission is far more scalable than a thermonuclear device. For example, LeMoyne and Mastroianni have conceptually demonstrated a pulsed propulsion configuration incorporating photofission through the use of an ultra-intense laser [2].

\subsection{Photofission}

The foundation to photofission is nuclear fission. Nuclear fission involves the splitting of a nucleus into two lesser atomic mass nuclei. Traditionally nuclear fission occurs as result of neutrons with appropriate kinetic energy interacting with the cross-section of the respective nucleus, such as uranium-235. A typical uranium-235 nuclear fission reaction is provided by Equation (1):

$$
{ }_{0}^{1} n+{ }_{92}^{235} U \rightarrow{ }_{56}^{141} \mathrm{Ba}+{ }_{36}^{92} \mathrm{Kr}+3{ }_{0}^{1} n
$$

[2] [7] [8].

In Equation (1) the fission of uranium-235 through one neutron yields barium-141, krypton-92, and three neutrons. A considerable quantity of energy is also yielded through the nuclear fission reaction. The energy yield can be derived based on the mass defect of the nuclear reaction [2] [7] [8].

By contrast photofission is initiated through the incidence of gamma radiation on the respective nucleus that satisfies the giant dipole resonance. The nucleus deforms because of oscillatory motion. With the nuclear deformation the Coulomb repulsions exceed the threshold of the strong nuclear force. As a result nuclear fission from photonic interaction ensues [9].

Photofission utilizing uranium-238 targets can be produced by ultra-intense lasers of the $10^{20} \mathrm{~W} / \mathrm{cm}^{2} \mathrm{range}$. The NOVA laser of Lawrence Livermore National Laboratory and VULCAN laser of Rutherford Appleton Laboratory have demonstrated the feasibility of photofission using uranium-238 [10]-[12]. Tabletop laser systems achieving the ultra-intense laser have been established for producing photofission of a uranium-238 target [12].

Schwoerer et al. exhibited photofission using a tabletop laser capable of achieving an intensity on the order of $10^{20} \mathrm{~W} / \mathrm{cm}^{2}$. The experimental process incorporated two sheets of tantalum before the uranium-238 target. The preliminary tantalum sheet approximately $50 \mu \mathrm{m}$ thick is used to generate a hot plasma. The subsequent tantalum sheet with a thickness of $1 \mathrm{~mm}$ functions as a bremsstrahlung converter as plasma accelerated electrons from the first sheet strike the second sheet [12].

There are many advantages to applying photofission for nuclear pulsed space propulsion. Photofission has been demonstrated by readily available sources, such as natural uranium isotopes, lead, and thorium [13] [14]. As opposed to a difficult to regulate neutron flux, photofission is controlled based on the activation of the ultra-intense laser, which can also be remote to the propulsion system [2]. 
LeMoyne and Mastroianni have previously established the photofission energy yield per input of ultra-intense laser energy ratio of $2.2 \times 10^{-6} \mathrm{~kJ}$ per $\mathrm{kJ}$ for characterizing the performance of photofission [2]. The established photofission energy yield per input of ultra-intense laser energy ratio assumes a photofission event produces 200 $\mathrm{MeV}$, approximating the energy yield of uranium-235 fission [2] [7]. Noted by Naik et al. the determination of photofission energy yield for uranium-238 is an evolving scientific subject pending convergence [15]. The photofission energy yield ratio developed by LeMoyne and Mastroianni utilizes the experimental findings that $7 \times$ $10^{4}$ fissions per joule of the ultra-intense laser are feasible [2] [10] [12].

\subsection{Ultra-Intense Laser}

The progressive evolution of laser technology derives from the influence of multiple industries, such as medicine, communications, computer/information systems, and manufacturing [7]. These multiple sources affecting the research and development of laser devices likely promotes considerable promise for the correlated evolution of ultra-intense laser systems. Ultra-intense lasers on the scale of $10^{20} \mathrm{~W} / \mathrm{cm}^{2}$ have generated sufficient bremsstrahlung photons to achieve photofission through institutions, such as Lawrence Livermore National Laboratory’s NOVA laser and Rutherford Appleton Laboratory's VULCAN laser [10]-[12].

A notable achievement is the demonstration of photofission through a tabletop laser by Schwoerer et al. A Jena 15 TW laser was incorporated into the experiment that applied titanium-sapphire, chirped pulse amplification, a repetition rate of $10 \mathrm{~Hz}$, and a $80 \mathrm{fs}$ duration 1.2J maximum energy threshold. With the area of the laser beam target bound to $5 \mu \mathrm{m}^{2}$ a laser intensity attaining the order of $10^{20} \mathrm{~W} / \mathrm{cm}^{2}$ was feasible [12].

\subsection{Magneto-Hydrodynamic Generator}

In order to provide an energy source for the ultra-intense laser an energy conversion source is applied to Project New Orion. The first stage launch vehicle to the proposed Project New Orion application has two operational capabilities: providing a preliminary velocity increment and providing an energy source to operate the ultra-intense laser. A magneto-hydrodynamic generator can provide an appropriate energy source by converting a portion the thermal/kinetic energy of the rocket thrust to electrical energy [16]-[18].

Magneto-hydrodynamic generator incorporates a strong magnetic field interacting with plasma, such as the propulsive flow of a rocket accelerating through an isentropic expanding diverging rocket nozzle, positioned between two conducting electrodes. This configuration results in the generation of an electric current with an associated voltage potential difference between the electrodes [16]-[18]. Because of the common features between a magneto-hydrodynamic generator and rocket propulsion system, such as high kinetic energy plasma rocket exhaust and cryogenic cooling for a superconducting magnet, magneto-hydrodynamic generators have been proposed as a electrical energy conversion system for rocket propulsion applications [17] [18].

Solbes et al. published a feasibility study for the conversion efficiency of a magneto-hydrodynamic generator. Although the study applied a storable propellant configuration, the results should be amenable for conservatively approximating the energy conversion efficiency of a cryogenic propulsion system. Solbes et al. applied a cryogenic magnet cooled by liquid nitrogen enabling a 5T magnetic field about the inlet channel. With a 95\% combustion efficiency producing a thermal power of 95MW, the magneto-hydrodynamic generator produces 10MW of extracted electrical power [18]. Based on the feasibility study published by Solbes et al. the electric energy conversion efficiency of the magneto-hydrodynamic generator will be conservatively scaled to $10 \%$ of the rocket propulsion thrust power. The electric conversion efficiency of the magneto-hydrodynamic generator would likely be highly especially with the amalgamation of cryogenic propellant densification.

\subsection{Cryogenic Propellant Densification}

In order to sustain the appropriate magnetic fields for a magneto-hydrodynamic generator a cryogenically superconducting magnetic system about the rocket nozzle is advised. Rocket nozzles are standardly cooled by a cryogenic loop through out the rocket nozzle [19] [20]. Such a cryogenic circuit can also be applied to sustaining a cryogenically superconducting magnet. In particular cryogenic propellant densification offers additional utility to the Project New Orion application.

Propellant densification of the cryogenic fuel (hydrogen) and cryogenic oxidizer (oxygen) incorporates lowering the temperatures of cryogenic propellant below their normal boiling point, such as achieving approx- 
imately a $10 \%$ increase in propellant density. The thermodynamic properties of propellant densification also significantly reduce boiling pressure [21]. Propellant densification is not only projected to advance the performance characteristics of the cryogenic first stage, but also advance the performance capabilities of the superconducting magnet for magneto-hydrodynamic generator energy conversion efficiency.

\subsection{First Stage Rocket Delivery System}

For the preliminary elucidation of the photofission attributes of the Project New Orion second stage, the first stage rocket delivery system will focus on the propulsion system characteristics, such as specific impulse. In essence the specific impulse represents the performance efficiency of a rocket propulsion system. A liquid hydrogen and liquid oxygen propulsion system offers the most considerable specific impulse of available conventional chemical rocket propulsion systems. As the envisioned first stage is intended to operate in a space environment, the specific impulse is based on vacuum conditions [19] [20]. Based on historical data for a liquid hydrogen and liquid oxygen propulsion system acquired by LeMoyne a conservative vacuum specific impulse of 450 seconds will be assumed for the pending analysis [22].

\section{Project New Orion: Conceptual Architecture}

Project New Orion entails the amalgamation of multiple high technology applications to provide a more feasible and scaleable pulsed nuclear propulsion system by contrast to the original Project Orion. Rather than a series of thermonuclear detonations of extraordinary proportion, a variable yield series of photofission pulsed nuclear propulsion events or a singular photofission pulsed nuclear propulsion eventwill be applied. Instead of risking the transport of an operational thermonuclear device, the photofission event can be safely initiated at a remote distance from an ultra-intense laser.

Ultra-intense lasers have been demonstrated to fit the geometric constraints of a tabletop laser. The ultra-intense laser for Project New Orion is anticipated to likewise satisfy relevant geometric constraints and associated mass constraints for the associated launch vehicle. The ultra-intense laser will provide an energy source derived from a magneto-hydrodynamic generator converting a portion of the propulsive thrust thermal/kinetic energy into electrical energy for operating the ultra-intense laser. The generated electrical energy implies a need for a temporary storage medium, such as a supercapacitor. The selection of an appropriate energy storage medium will be a subject of pending refinement of the Project New Orion endeavor, such as further convergence of the design for the first stage propulsion system.

Cyrogenic propellant densification will be applied to enable optimal performance capabilities of the superconducting magnet for the magneto-hydrodynamic generator and first stage propulsion system. The first stage will incoroprate a cryogenic liquid hydrogen liquid oxygen propulsion system. Alternative configurations for Project New Orion are either the incorporation of the Project New Orion propulsion system into a second stage or a disparate spacecraft system relative to the first stage propulsion system.

\section{Project New Orion: Fundamental Performance Analysis}

The fundamental performance analysis of Project New Orion will pertain to preliminary analysis of the photofission second stage with associated relevant analysis for the liquid hydrogen-oxygen first stage. A more detailed definition of the liquid hydrogen-oxygen first stage will be incorporated into later and more detailed analysis of Project New Orion. Based on the prescribed performance parameters of the first stage, which will be ignited in a space vacuum environment, the relevant performance parameters of the photofission second stage are derived. The following parameters are assumed for the fundamental performance analysis of Project New Orion:

- Vacuum specific impulse of the hydrogen-oxygen propulsion system: 450 seconds.

- Mass flow of the hydrogen-oxygen propulsion system: $100 \mathrm{~kg} / \mathrm{seconds}$.

- Thrust duration of the first stage propulsion system: 1000 seconds.

- Magneto-hydrodynamic generator electric energy conversion efficiency: $10 \%$.

- Photofission energy yield per input of ultra-intense laser energy ratio: $2.2 \times 10^{-6} \mathrm{~kJ} \cdot \mathrm{per} \cdot \mathrm{kJ}$.

- Mass of the Project New Orion spacecraft with nozzle-like pusher assembly: $1 \mathrm{~kg}$.

- Electrical energy to ultra-intense laser energy efficiency: $10 \%$.

- Kinetic energy efficiency of Project New Orion spacecraft with hemispherical nozzle-like pusher assembly: $50 \%$. 
First the thrust power of the first stage is derived under the assumption that the propulsion system nozzle expands the propulsive flow to a pressure equivalence of the ambient vacuum space environment. The vacuum specific impulse Equation (2) provides the basis for deriving the exhaust velocity of the rocket propulsion exhaust.

$$
\begin{gathered}
I_{s p}=\frac{F}{\dot{m} g}=\frac{\dot{m} v}{\dot{m} g}=\frac{v}{g} \rightarrow v=g \cdot I_{s p} \\
I_{s p}: \text { Specific Impulse (seconds) } \\
F: \text { Thrust }(\text { Netwons) } \\
\dot{m}: \text { Mass Flow }(\mathrm{kg} / \mathrm{s}) \\
g: \operatorname{Gravity}\left(\mathrm{m} / \mathrm{s}^{2}\right) \\
v: \operatorname{Velocity}(\mathrm{m} / \mathrm{s})
\end{gathered}
$$

[19] [20].

Based on fundamental performance analysis of the first stage propulsion system, the exhaust velocity is calculated to be $4410 \mathrm{~m} / \mathrm{s}$. With the exhaust velocity obtained thrust power per Equation (3) is determined.

$$
P=\frac{1}{2} \dot{m} v^{2}
$$

\section{$P:$ Thrust Power $(W)$}

[19] [20].

The thrust power is calculated to be $9.7 \times 10^{8} \mathrm{~W}$. The duration of the first stage rocket operation is 1000 seconds. Therefore over the duration of the first stage activity the energy derived from the thrust is $9.7 \times 10^{11} \mathrm{~J}$. Based on the magneto-hydrodynamic generator electric energy conversion efficiency of $10 \%, 9.7 \times 10^{10} \mathrm{~J}$ of electrical energy will be available, which with a $10 \%$ energy converision efficiency to the ultra-intense laser system provides the ultra-intense laser with $9.7 \times 10^{9} \mathrm{~J}$ of laser energy.

Using the photofission energy yield per input of ultra-intense laser energy ratio of $2.2 \times 10^{-6} \mathrm{~kJ}$ per kJ with $9.7 \times 10^{9} \mathrm{~J}$ of laser energy. The photofission energy yields $2.1 \times 10^{4} \mathrm{~J}$ of energy. Using a $50 \%$ kinetic energy efficiency of the Project New Orion spacecraft with hemispherical nozzle-like pusher assembly $1.1 \times 10^{4} \mathrm{~J}$ of kinetic energy is imparted on the $1 \mathrm{~kg}$ Project New Orion spacecraft, which produces a velocity increment of 150 $\mathrm{m} / \mathrm{s}$ using photofission through an ultra-intense laser. This velocity increment could be achieved either from a series of pulses summing to the equivalence of the precribed energy constraints or one singular pulse to the equivalence of the prescibed energy constraints.

\section{Conclusion}

Project New Orion offers a feasible pathway for the progressive research, test, and evaluation of pulsed nuclear propulsion configurations. Photofission through the application of an ultra-intense laser enables a series or singular pulse of nuclear yield to impart a velocity increment on a small spacecraft. The viability of Project New Orion is demonstrated through fundamental performance analysis. The historical origins stem from the efforts of Project Orion, which advocated the use of thermonuclear devices for providing a substantial velocity increment to a respective spacecraft. However, the magnitude of Project Orion in essence precludes the probability of progressive research, development, testing, and evaluation. With the advances of associated technologies Project New Orion may offer a reasonable pathway for the endeavor of pulsed nuclear propulsion. An energy source for the ultra-intense laser producing photofission is enabled through a magneto-hydrodynamic generator that converts a portion of the thermal/kinetic energy from the first stage rocket exhaust to electrical energy. Cryogenic propellant densification provides synergistic advantage for the magneto-hydrodynamic generator superconducting magnet and first stage propulsion system. Fundamental performance analysis of Project New Orion establishes the capability to impart a meaningful velocity increment on a small spacecraft through ultra-intense laser derived photofission. 


\section{References}

[1] LeMoyne, R. and Mastroianni, T. (2014) Fundamental Architecture and Analysis of an Antimatter Ultra-Intense Laser Derived Pulsed Space Propulsion System. Journal of Applied Mathematics and Physics, 2, 10-18. http://dx.doi.org/10.4236/jamp.2014.25002

[2] LeMoyne, R. and Mastroianni, T. (2015) Fundamental Architecture and Performance Analysis of Photofission Pulsed Space Propulsion System Using Ultra-Intense Laser. Journal of Applied Mathematics and Physics, 3, 436-442. http://dx.doi.org/10.4236/jamp.2015.34055

[3] Nance, J.C. (1965) Nuclear Pulse Propulsion. IEEE Transactions on Nuclear Science, 12, 177-182. http://dx.doi.org/10.1109/TNS.1965.4323511

[4] Dyson, F.J. (1968) Interstellar Transport. Physics Today, 21, 41-45. http://dx.doi.org/10.1063/1.3034534

[5] Schmidt, G.R., Bunometti, J.A. and Morton, P.J. (2000) Nuclear Pulse Propulsion—Orion and beyond. Proceedings of 36th AIAM/ASME/SAE/ASEE Joint Propulsion Conference \& Exhibition, Huntsville, July 2000. http://dx.doi.org/10.2514/6.2000-3856

[6] Always/Never. https://share.sandia.gov/news/resources/news_releases/always_never/\#.VmZ48-MrIcg

[7] Serway, R.A., Moses, C.J. and Moyer, C.A. (1989) Modern Physics. Saunders College, Philadelphia.

[8] Serway, R.A. (1990) Physics for Scientists and Engineers. Saunders College, Philadelphia.

[9] Kavouras, J.G. (2014) Detection and Simulation of Delayed $\gamma$-Rays from Photofission. Master's Thesis, Department of Civil and Environmental Engineering (Nuclear Engineering), University of Utah, Salt Lake City.

[10] Cowan, T.E., Hunt, A.W., Phillips, T.W., Wilks, S.C., Perry, M.D., Brown, C., Fountain, W., Hatchett, S., Johnson, J., Key, M.H., Parnell, T., Pennington, D.M., Snavely, R.A. and Takahashi, Y. (2000) Photonuclear Fission from High Energy Electrons from Ultraintense Laser-Solid Interactions. Physical Review Letters, 84, 903-906. http://dx.doi.org/10.1103/PhysRevLett.84.903

[11] Ledingham, K.W., Spencer, I., McCanny, T., Singhal, R.P., Santala, M.I., Clark, E., Watts, I., Beg, F.N., Zepf, M., Krushelnick, K., Tatarakis, M., Dangor, A.E., Norreys, P.A., Allott, R., Neely, D., Clark, R.J., Machacek, A.C., Wark, J.S., Cresswell, A.J., Sanderson, D.C. and Magill, J. (2000) Photonuclear Physics When a Multiterawatt Laser Pulse Interacts with Solid Targets. Physical Review Letters, 84, 899-902. http://dx.doi.org/10.1103/PhysRevLett.84.899

[12] Schwoerer, H., Ewald, F., Sauerbrey, R., Galy, J., Magill, J., Rondinella, V., Schenkel, R. and Butz, T. (2003) Fission of Actinides Using a Tabletop Laser. EPL (Europhysics Letters), 61, 47-52. http://dx.doi.org/10.1209/epl/i2003-00243-1

[13] Cetina, C., Berman, B.L., Briscoe, W.J., Cole, P.L., Feldman, G., Heimberg, P., Murphy, L.Y., Philips, S.A., Sanabria, J.C., Crannell, H., Longhi, A., Sober, D.I. and Kezerashvili, G.Y. (2000) Photofission of Heavy Nuclei at Energies up to $4 \mathrm{GeV}$. Physical Review Letters, 84, 5740-5743. http://dx.doi.org/10.1103/PhysRevLett.84.5740

[14] Cetina, C., Heimberg, P., Berman, B.L., Briscoe, W.J., Feldman, G., Murphy, L.Y., Crannell, H., Longhi, A., Sober, D.I., Sanabria, J.C. and Kezerashvili, G.Y. (2002) Photofission of Heavy Nuclei from 0.2 to $3.8 \mathrm{GeV}$. Physical Review C, 65, Article ID: 044622. http://dx.doi.org/10.1103/PhysRevC.65.044622

[15] Naik, H., Carrel, F., Kim, G.N., Laine, F., Sari, A., Normand, S. and Goswami, A. (2013) Mass Yield Distributions of Fission Products from Photo-Fission of 238U Induced by 11.5 - 17.3 MeV Bremsstrahlung. The European Physical Journal A, 49, 1-23. http://dx.doi.org/10.1140/epja/i2013-13094-7

[16] Ayeleso, A.O., Kahn, M.T.E. and Raji, A.K. (2015) Plasma Energy Conversion System for Electric Power Generation. Proceedings of 2015 International Conference on the Industrial and Commercial Use of Energy (ICUE), Cape Town, August 2015, 206-211. http://dx.doi.org/10.1109/ICUE.2015.7280270

[17] He, Z., Huam, L.Z. and Yao, F.G. (1994) Research on a Super-Small-Scale MHD Power System. IEEE Transactions on Energy Conversion, 9, 186-191. http://dx.doi.org/10.1109/60.282491

[18] Solbes, A., Weede, J., Hession, R. and Mitchell, P. (1989) Design of a Ten Megawatt Rocket Driven Disk MHD Generator. Proceedings of the 24th Intersociety Energy Conversion Engineering Conference, Washington DC, August 1989, 1073-1078. http://dx.doi.org/10.1109/IECEC.1989.74600

[19] Sutton, G.P. (1992) Rocket Propulsion Elements. Wiley-Interscience, New York.

[20] Sutton, G.P. (2006) History of Liquid Propellant Rocket Engines. AIAA, Reston. http://dx.doi.org/10.2514/4.868870

[21] LeMoyne, R. (2008) Fundamental Analysis of Cryogenic Propellant Densification. Proceedings of 15th AIAA International Space Planes and Hypersonic Systems and Technologies Conference, Dayton, April 2008. http://dx.doi.org/10.2514/6.2008-2500

[22] LeMoyne, R. (2008) Multidisciplinary Cost and Performance Optimization of a Two Stage Liquid Propulsion Based Launch Vehicle. Proceedings of 15th AIAA International Space Planes and Hypersonic Systems and Technologies Conference, Dayton, April 2008. http://dx.doi.org/10.2514/6.2008-2642 